\title{
Avaliação da influência das tensões de barra na previsão de cargas via redes neurais
}

\author{
Evaluation of the influence of the bus-bar voltage profiles on demand forecasting by using neural \\ networks \\ Evaluación de la influencia de los voltajes de barra en el pronóstico de la demanda mediante el uso
}

de redes neuronales

Recebido: 22/09/2021 | Revisado: 28/09/2021 | Aceito: 30/09/2021 | Publicado: 02/10/2021

Amanda Thais dos Reis Fernandes
ORCID: https://orcid.org/0000-0002-3388-9681
Universidade Federal do Amapá, Brasil
E-mail: fernandes@ieee.org
Jean Lucas Tourinho Fonseca
ORCID: https://orcid.org/0000-0001-9927-6274
Universidade Federal do Amapá, Brasil
E-mail: tourinho@ieee.org
Iuri Leno Pereira da Silva
ORCID: https://orcid.org/0000-0003-2534-9906
Universidade Federal do Amapá, Brasil
E-mail: iuri.unifap2016@gmail.com
Piedy Del Mar Agamez Arias
Oriel
ORCID: https://orcid.org/0000-0002-5397-0989
INYCIA Reserch Group, Colombia
E-mail: piedyagamez@gmail.com
Rodrigo Andrade Ramos
ORCID: https://orcid.org/0000-0002-2240-1150
Universidade de São Paulo, Brasil
E-mail: rramos@usp.edu.br
Werbeston Douglas de Oliveira
ORCID: https://orcid.org/0000-0002-4566-6290
Universidade Federal do Amapá, Brasil
E-mail: wdoliveira@unifap.br

\section{Resumo}

A previsão de carga de curtíssimo prazo permite aos engenheiros de operação um despacho econômico e seguro do sistema elétrico, além de ajudar na composição dinâmica de preços no mercado de energia. Diversas metodologias como análise de regressão, series temporais, abordagens de aprendizado de máquina, métodos de aprendizado profundo e inteligência artificial tem sido usadas para prever a carga. Mas, diversos fatores externos tornam a previsão uma tarefa mais complexa do que aparenta ser inicialmente. Assim, as redes neurais artificiais têm-se apresentado como uma técnica de inteligência computacional capaz de lidar com o problema da previsão de carga com grande precisão. Neste contexto, este trabalho visa avaliar o impacto do perfil de tensão das barras do sistema elétrico sobre a previsão da carga. Para isto, foi proposto estudar três arranjos de bases de dados ((1) dados normalizados do histórico de carga; (2) dados normalizados do histórico de carga e tensão nas barras de carga; e, (3) dados normalizados do histórico de carga, tensão nas barras de carga e sazonalidade destes) para treinar nove redes neurais do tipo MLP de duas camadas. A abordagem proposta é avaliada com base em dados exportados pelo estimador de estados da rede de uma grande empresa da região norte do Brasil. Os resultados indicam que, segundo os valores de MSE e MAPE obtidos, todas as redes neurais avaliadas atingem a previsão esperada. No entanto, o melhor desempenho foi alcançado com o arranjo que considerou uma base de dados a partir do histórico de carga normalizado e tensão nas barras de carga.

Palavras-chave: Previsão de carga; Redes neurais; Tensão nas barras, Histórico de carga; Curtíssimo prazo.

\section{Abstract}

The very short-term load forecasting allows operation engineers an economic and safe dispatch of the power system while dynamically contributes to the prices in the energy market. Several methodologies such as regression analysis, time series, machine learning approaches, deep learning methods, and artificial intelligence have been used to forecast load. However, several external factors become the forecasting a more complex task than it initially appears to be. For this reason, neural networks have been presented as a computational intelligence technique capable of dealing with the load forecasting problem with great precision. In this context, this work aims to evaluate the impact of voltage profiles of the power system bus on the load forecasting. For this, it was proposed to study three database arrangements ((1) 
normalized load historical data; (2) normalized load historical data and voltage profile in load bars; and, (3) normalized load historical data, voltage profile in load bus and seasonality of these loads) to train nine neural networks of the MLP type with two layers. The proposed approach is evaluated based on data obtained from the state estimator of a network of a large company in the northern region of Brazil. The results show that, according to the MSE and MAPE values obtained, all the neural networks evaluated achieve a forecasting of the load as expected. However, the best performance was achieved with the arrangement that considered a database that records a normalized load historical data and voltage profile in load bus.

Keywords: Load forecasting; Very short-term; Neural networks; Load historical data; Voltage profile.

\section{Resumen}

El pronóstico de demanda de muy corto plazo permite a los ingenieros de operación un despacho económico y seguro del sistema eléctrico, además de ayudar en la composición dinámica de los precios en el mercado de energía. Varias metodologías como análisis de regresión, series temporales, enfoques de aprendizaje de máquina, métodos de aprendizaje profundo e inteligencia artificial han sido usadas para pronosticar la demanda. Sin embargo, varios factores externos tornan el pronóstico una tarea más compleja de lo que inicialmente aparenta ser. Es por ello, que las redes neuronales se han presentados como una técnica de inteligencia computacional capaz de lidiar con el problema de la previsión de carga con una grande precisión. En este contexto, este trabajo pretende evaluar el impacto de los perfiles de tensión de las barras del sistema eléctrico sobre el pronóstico de la demanda. Para ello, fue propuesto estudiar tres arreglos de bases de datos ((1) datos normalizados del histórico de carga; (2) datos normalizados del histórico de carga y tensión en las barras de carga; e, (3) datos normalizados del histórico de carga, tensión en las barras de carga y estacionalidad de estas) para entrenar nueve redes neuronales del tipo MLP con dos capas. El enfoque propuesto es evaluado con base en datos exportados por el estimador de estado de una red de una gran empresa de la región norte de Brasil. Los resultados indican que, según los valores de MSE y MAPE obtenidos, todas las redes neuronales evaluadas consiguen el pronóstico esperado. No obstante, el mejor desempeño fue alcanzado con el arreglo que consideró una base de datos a partir del histórico de carga normalizado y la tensión en las barras de carga.

Palabras clave: Pronóstico de demanda; Muy cirto plazo redes neuronales; Histórico de carga; Perfiles de tensión.

\section{Introdução}

Em um Sistema Elétrico de Potência (SEP) é fundamental que seja conhecido as cargas que serão atendidas, pois com base na carga prevista diversos estudos são desenvolvidos, por exemplo: análise de segurança, despacho econômico, estudos de estabilidade transitória e composição no preço de mercado (Antunes, 2013; Oliveira, 2012). Tendo em vista que a carga é uma variável tão importante e não determinística é indispensável que se possa ter uma estimativa segura de suas características (Prito, et al., 2006).

Para realizar a previsão de carga é preciso que sejam identificados padrões de consumo, isto pode ser feito com base no histórico de consumo. Originalmente o problema foi resolvido de forma matemática e estatística através de técnicas tais como as séries numéricas, que fornecem bons resultados, mas utilizam processos complexos e de difícil modelagem (Guirelli, 2006). Isto é, há diversos fatores externos, tais como temperatura, estações do ano, hábitos de consumo, eventos esportivos, entre outros, que tornam a previsão uma tarefa complicada.

No entanto, atualmente os modelos de previsão têm conseguido alcançar baixos valores percentuais de erro. O que se dá devido ao uso de métodos de Inteligência Artificial (do Inglês, Intelligence Artificial - IA), dentre esses métodos pode-se destacar as Redes Neurais Artificiais (RNA). Conceitualmente, uma rede neural artificial é um dispositivo tanto capaz de processar informação de forma distribuída quanto de incorporar conhecimento através de exemplos. Trata-se, portanto, de um processador capaz de extrair conhecimento experimental disponibilizando-o para uso prático (Zanini, 2004). Por exemplo, Caires (2021) usa RNA previsão de demanda brasileira de biodiesel, que resultou em aumento da demanda do mesmo. Pessoa (2021) mostra que RNA é capaz de predizer a capacidade de carga de estacas cravadas para fundações.

A principal vantagem de uma ANN, segundo Kovàcs (1997), é a sua capacidade de aproximar relações funcionais, particularmente quando as relações não são bem definidas e/ou são não-lineares, o que torna difícil a utilização de métodos convencionais para a tentativa de prever as variações futuras dessas relações. Ou seja, diversos problemas complexos podem ser abordados por RNA. 
Ainda que os métodos de IA aparentam ser mais simples, estes possuem problemas próprios, é difícil determinar quais dados e variáveis são importantes para o sistema de previsão, bem como a parametrização da rede nas etapas de treinamento. Para melhorar suas redes neurais, Singh (2017) usa diferentes perfis de carga para dias da semana e fins de semana para melhorar os resultados da previsão. Chafi (2021) usa otimização por enxame de partículas para o ajuste de parâmetros da RNA para previsão de cargas, enquanto uma revisão da literatura é realizada por Baliyan (2015), onde verificasse que diferentes estruturas de RNAs são utilizadas para previsão de carga.

Por esse motivo, este trabalho vem com o intuito de realizar comparações entre três arranjos de RNA para previsão de carga de curtíssimo prazo, com entradas para treinamento diferentes, de modo a definir qual destes arranjos resulta em menor erro de previsão. Os arranjos básicos serão compostos de: 1) Histórico de carga; 2) Histórico de carga e tensão nas barras de carga; e 3) Histórico de carga, valor de tensão nas barras de carga e sazonalidade destes. Neste trabalho será utilizado uma RNA do tipo Multi Layer Perceptron (MLP) de duas camadas com neurônios ocultos para previsão de carga de curtíssimo prazo.

A escolha de considerar a tensão nas barras de carga é devido ao fato de que as cargas podem variar de acordo com a tensão a qual está submetida, conforme descrito pelo modelo polinomial (Kundur, 1994). Como trata-se de um estudo para previsão de curtíssimo prazo, uma pequena mudança no valor de tensão pode ter efeito na demanda. As equações abaixo apresentam o modelo polinomial.

$$
\begin{aligned}
& P=P_{0}\left[p_{1} \times V^{2}+p_{2} V+p_{3}\right] \\
& Q=Q_{0}\left[q \times V^{2}+q_{2} V+q_{3}\right]
\end{aligned}
$$

\section{Referencial Teórico}

\subsection{Previsão de carga}

Segundo Morettin e Toloi (2006) a palavra previsão sugere que se quer ver uma coisa antes que ela exista. Os autores destacam que a previsão não constitui um fim em si, mas apenas um meio de fornecer informações para uma consequente tomada de decisões, visando a determinados objetivos.

Os modelos de previsão são amplamente utilizados em várias áreas do conhecimento, como previsão de mercado de ações (Vieira \& Thomé, 2000) e predição de produtividade de milho (Leal, et al., 2015), sendo um mecanismo importante em diversas aplicações. Esta função de previsão se faz necessária no segmento dos sistemas elétricos de potência, pois a antecipação da demanda energética é fundamental para os SEPs, uma vez que as predições proporcionam base para a tomada de decisões no que se refere às atividades de operação e planejamento. Uma previsão quando empregue na prática varia muito, podendo ser simples e intuitiva ou mais quantitativa e complexa.

Para realizar uma previsão de carga define-se as variáveis que podem influenciar no valor da demanda do sistema, juntamente com a possível variação desses fatores. Definida essas variáveis, utiliza-se seus dados históricos com o objetivo de identificar e determinar o comportamento/padrão da demanda do sistema.

As previsões de carga podem ter um horizonte de curtíssimo, curto, médio e longo prazo. Na previsão a curtíssimo prazo, a previsão é feita para alguns minutos até uma hora à frente, esta é utilizada em sistemas de tempo real. A previsão a curto prazo varia de algumas horas até uma semana à frente, é usada na programação da manutenção e auxílio à operação do sistema. Na previsão de médio prazo, a previsão é realizada de semanas até meses à frente, é empregada para intercâmbio de entre as concessionárias e no planejamento da produção. A previsão de longo prazo é para anos à frente e é utilizada para o planejamento estratégico e para prever possíveis construções de estruturas geradoras de energia. 
Ferreira (2020) ressalta que, a imprecisão nas previsões pode resultar em um considerável aumento nos gastos operacionais desses sistemas, causando grandes prejuízos à companhia elétrica. Desse modo, a predição da carga a ser atendida deve ser viabilizada de maneira ágil, rápida, precisa e confiável.

Tradicionalmente, utilizam-se abordagens clássicas que exigem uma modelagem matemática e estatística para a previsão de carga, como: modelo de ARIMA (Box \& Jenkins, 1976; Cho, et al., 1995), Regressão Linear (Amral, et al., 2007; Hong et al., 2010), Alisamento Exponencial (Christiaanse, 1971; Taylor, 2012), entre outros. Os métodos estatísticos de previsão podem ser divididos em métodos baseados em séries temporais (modelo ARIMA) e regressão (regressão linear e alisamento exponencial).

Uma série temporal é um conjunto de observações feitas sequencialmente ao longo do tempo (Box \& Jenkins, 1976). Uma das características mais importantes deste tipo de dados é que as observações vizinhas são correlacionadas e há interesse na análise e modelagem desta correlação. Segundo Silva (2010), as séries temporais podem ser classificadas como discretas, contínuas, determinísticas, estocásticas, multivariadas e multidimensionais.

Ehlers (2009) destaca que, os principais objetivos em se estudar séries temporais podem ser os seguintes: (1) Descrição, para descrever propriedades da série; (2) Explicação, de modo que se possa usar a variação em uma série para explicar a variação em outra série; (3) Controle, os valores da série temporal medem a "qualidade" de um processo de manufatura e o objetivo é o controle do processo; e (4) Predição, isto é, predizer valores futuros com base em valores passados.

Os modelos de regressão avaliam a carga a ser prevista como uma combinação linear da temperatura, de valores passados da carga entre outras variáveis (Almeida, 2013). Os modelos de regressão são construídos com os objetivos: (1) Predição, pois espera-se que a variável de saída seja explicada pelas variáveis de entrada; (2) Escolha de variáveis, a análise de regressão pode auxiliar na seleção de variáveis importantes para o problema; (3) Inferência, o modelo de regressão pode também ser utilizado para realizar testes de hipóteses e intervalos de confiança.

Apesar dos métodos estatísticos serem amplamente desenvolvidos, esses apresentam dificuldades. Pois, são muitas as variáveis que afetam a demanda de energia em um sistema, tornando a previsão bastante complexa. Neste sentido, os métodos baseados em inteligência artificial vêm se mostrando uma boa ferramenta para previsão de carga, uma vez que não é necessária uma elaboração de modelos matemáticos complexos (Brito et al., 2006; Antunes, 2013; Pessanha \& Justino, 2013).

A Inteligência Artificial pode ser entendida como o conjunto de técnicas e metodologias de programação usadas para tentar resolver problemas de forma mais próxima possível de um ser humano (Almeida, 2013). Dentre os métodos baseados em inteligência artificial se destacam as Redes Neurais Artificiais e Lógica Fuzzy. Há também modelos híbridos que combinam dois ou mais tipos de previsão.

\subsection{Redes Neurais Artificiais}

Uma ANN possui algumas características de desempenho em comum com as redes neurais biológicas (do Inglês, Biological Neural Network - BNN). De acordo com Haykin (1999), essas se assemelham em dois aspectos: (1) Ambas extraem conhecimento do ambiente através de um processo de aprendizagem ou treinamento; e (2) As forças de conexão entre os neurônios, conhecidos como pesos sinápticos, são utilizados para armazenar o conhecimento adquirido.

As Figuras 1 e 2 mostram a representação de um neurônio biológico e um neurônio artificial, respectivamente. A Tabela 1 apresenta semelhanças entre ambos. 
Figura 1. Rede neural biológica.

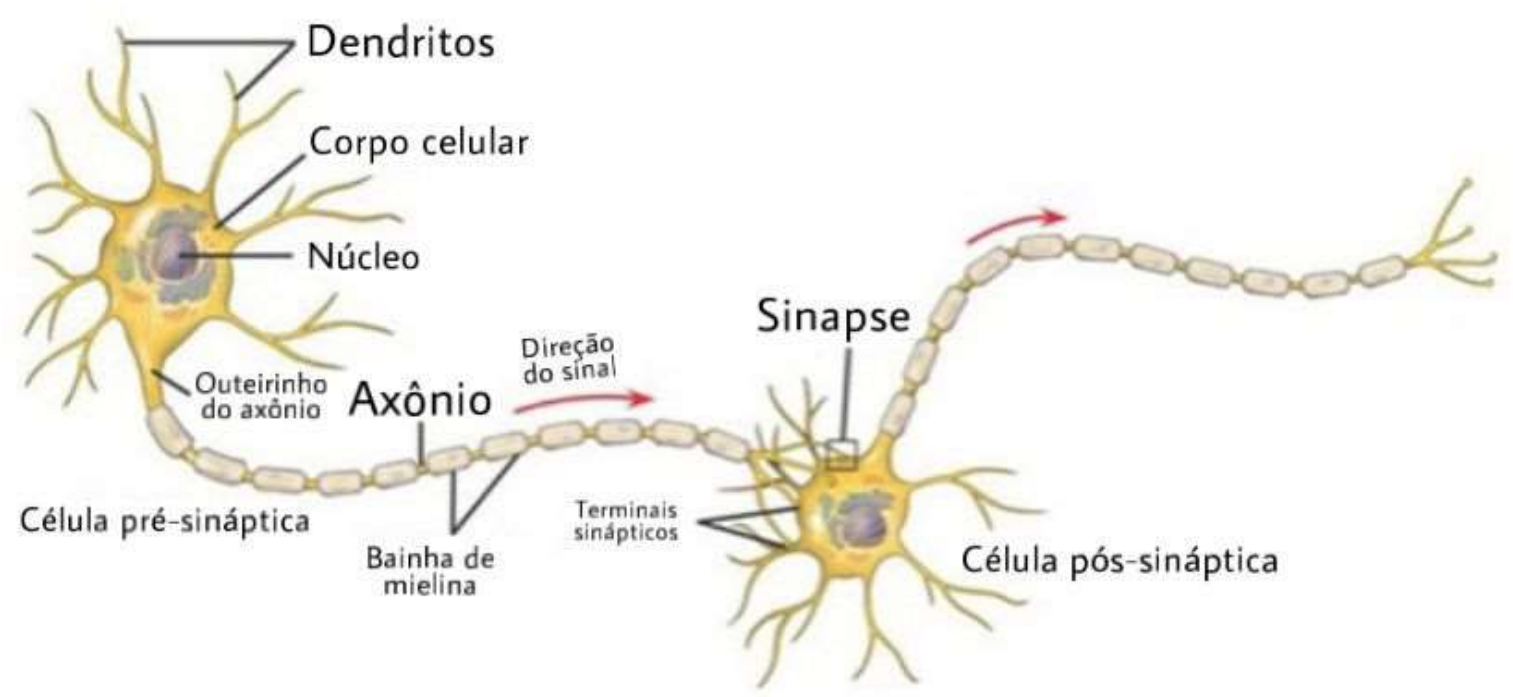

Fonte: Adaptado de Chaika (2020).

Figura 2. Representação de uma ANN.

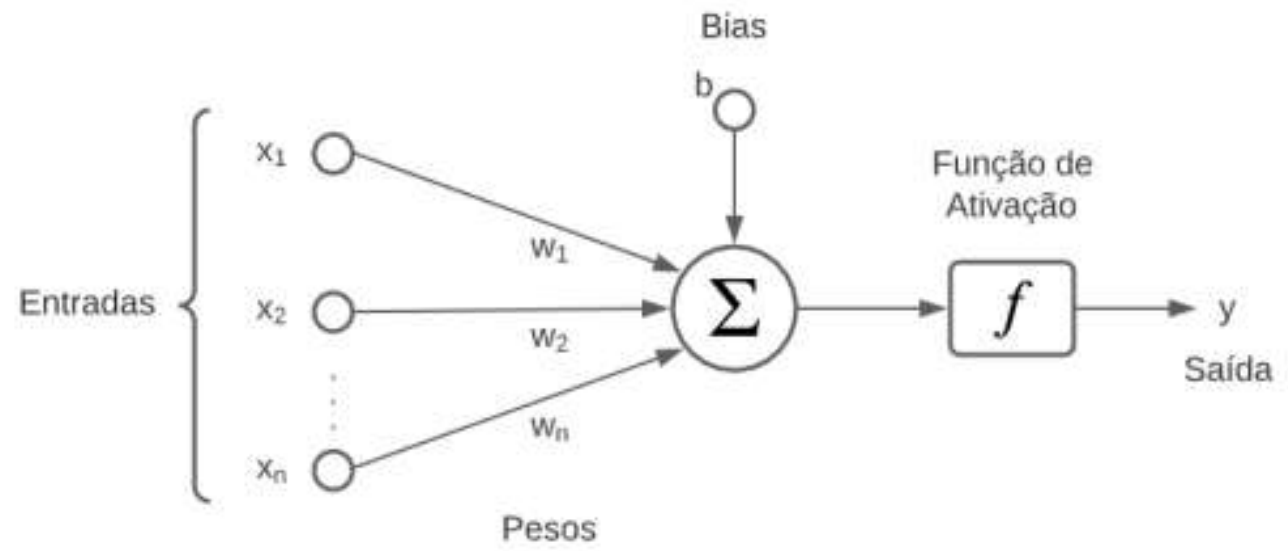

Fonte: Autores.

Tabela 1. Similaridade entre rede neural biológica e artificial.

\begin{tabular}{|c|c|}
\hline BNN & ANN \\
\hline Dendritos & Entradas \\
Sinapse & Pesos \\
Axônio & Saída \\
\hline
\end{tabular}

Fonte: Shaikh (2020).

Uma ANN é composta basicamente por um vetor de entrada, função de ativação, neurônios e pesos dos neurônios. O processamento da informação ocorre com o auxílio dos neurônios, cada neurônio possui uma função de ativação, os sinais são propagados de um elemento a outro através de conexões que possuem um peso associado.

O treinamento da ANN consiste em ajustar o peso associado a cada conexão entre os neurônios até que as saídas computadas para cada conjunto de entradas de dados sejam o mais próximo possível das saídas de dados experimentais. Em outras palavras, o erro entre a saída e a saída desejada é minimizado, possivelmente para zero. (Ettaouil \& Ghanou, 2009). 
Há diversas arquiteturas de ANN, sendo as mais utilizadas para previsão de cargas: Multilayer Perceptron (MLP) e a Radial Basis Functions (RBF). Neste trabalho será utilizada a arquitetura MLP.

A aplicação de ANNs para resolução de problemas de predição em séries temporais tem se mostrado eficiente, com baixos valores de erros nas previsões. Segundo Highley (1993), as ANNs combinam as características inerentes às técnicas padrões para resolução de séries temporais, mas com a vantagem de não ser necessário o conhecimento das relações funcionais, pois as ANNs são treinadas e não programadas.

\subsubsection{Perceptron simples}

O perceptron, modelo criado por Frank Rosenblatt, é um dos primeiros modelos de aprendizagem em machine learning. Segundo Adeli e Yeh (1989), um perceptron é definido como uma entidade de quatro tuplas que pode responder "sim" ou "não" no domínio do problema.

Figura 3. Perceptron simples

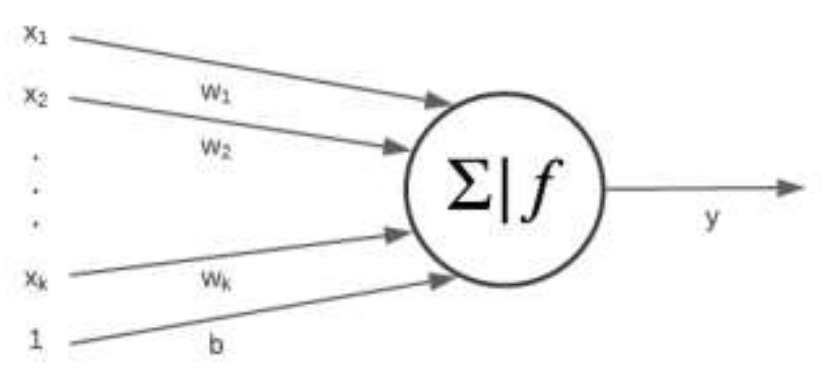

Fonte: Autores.

A Figura 3 apresenta um perceptron simples. Pode-se observar que um perceptron recebe entradas $x_{i}$, onde cada uma terá um peso $w_{i}$ e será feita a soma junto com um bias, $b$, que permite uma melhor adaptação ao conhecimento que será fornecido.

$$
s=\sum_{i=1}^{k}\left(x_{i} \times w_{i}\right)+b
$$

Essa soma então é aplicada em uma função de ativação que retorna o valor de saída, isto é, $f(s)=y$. Com o valor de saída da ANN, $y(t)$, e o valor de saída desejada, $d(t)$, é então atualizado os pesos de acordo com o erro entre o desejado e o obtido.

$$
w(t+1)=w(t)+\eta(\mathrm{d}(\mathrm{t})-y(t))
$$

Onde $\eta \in(0,1)$ representa uma taxa de aprendizado. Com o peso final definido no treinamento, pode-se fazer o teste (com diferentes entradas) para avaliar a ANN.

\subsubsection{Perceptron multicamadas}

Uma rede neural com somente um neurônio a limita muito, a utilização de combinação de neurônios em camadas pode tornar a ANN mais precisa, esse tipo de arquitetura é conhecido como MLP. A Figura 4 apresenta uma MLP. 
O aprendizado desse tipo de rede neural é geralmente realizado através do algoritmo Backpropagation (abreviação de backward propagation errors), onde o erro da saída final é calculado e é propagado da camada de saída até a camada de entrada, passando pelas camadas ocultas, isto é, de trás pra frente. Ao final deste processo os pesos são ajustados. Neste trabalho foi utilizada a formulação descrita em Ramchoun, Idrissi, Ghanou e Ettaouil (2015).

Figura 4. Perceptron multicamada.

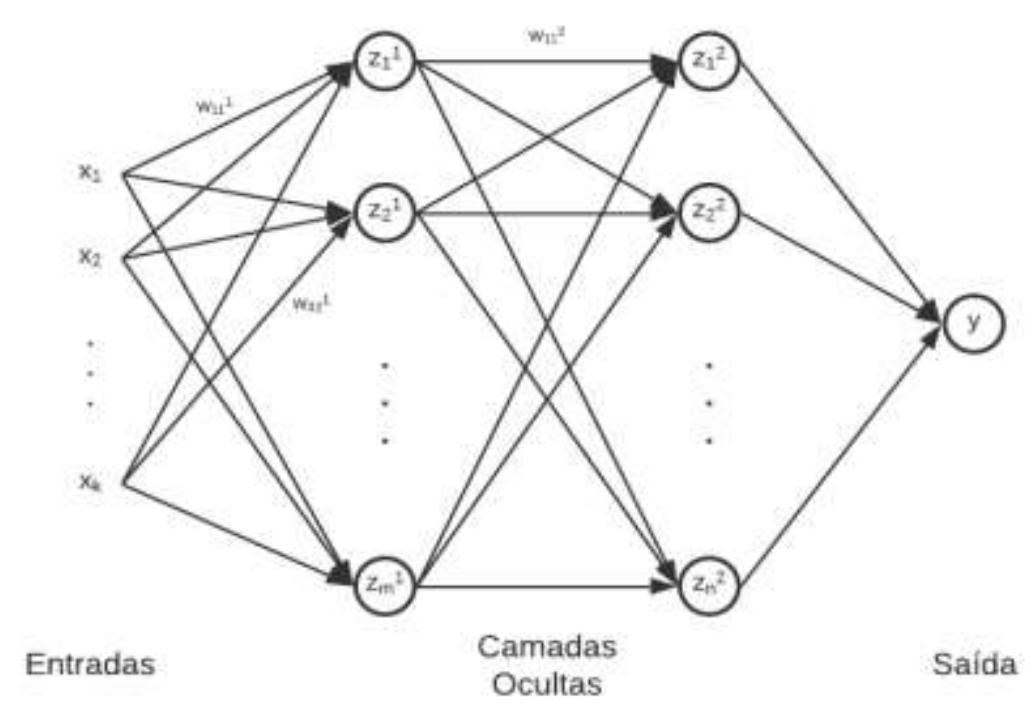

Fonte: Autores.

\section{Metodologia}

O presente trabalho adota uma metodologia de natureza explicativa, onde se pretende verificar a influência da tensão das barras do sistema na carga a ser prevista. Trata-se de um estudo de caráter quantitativo, para isso realizou-se pesquisa bibliográfica a fim do levantamento e análise de dados. Assim, foi possível a utilização de dados exportados pelo estimador de estados da rede de uma grande empresa da região norte do Brasil para validação dos resultados obtidos (Estrela, 2018; Pereira, et al., 2018)

Com o intuito de prover a antecipação da demanda de cargas elétricas, o algoritmo de treinamento escolhido foi o backpropagation de Levenberg-Marquardt. Este método procura o mínimo local em uma função e converge mais rapidamente do que um algoritmo genético.

Para o desenvolvimento do projeto é necessário de um ou mais banco de dados para o treino das ANN, os dados utilizados neste estudo foram extraídos do estimador de estados da rede de uma empresa de eletricidade do norte do Brasil.

Após um tratamento nos dados obtidos, quatro bancos de dados estavam disponíveis para serem utilizados, sendo histórico de carga, sazonalidade do histórico de carga, tensão nas barras e sazonalidade das tensões. Sazonalidade é a presença de variações que ocorrem em intervalos regulares específicos, por exemplo, consumo elevado nos meses de inverno devido a temperatura. Neste trabalho a sazonalidade pode ser obtida após um tratamento nos dados realizado com o auxílio do software Pycharm.

Realizou-se combinações dos bancos de dados para o treino das ANNs, dando origem aos arranjos abaixo.

Arranjo 1: Composto pelo histórico de carga normalizado;

Arranjo 2: Composto pelo histórico de carga normalizado e tensão nas barras de carga;

Arranjo 3: Histórico de carga normalizado, valor de tensão nas barras de carga e sazonalidade destes. 
Optou-se por treinar as redes neurais com o histórico de carga normalizado pelo fato de que a tensão nas barras de carga estava no sistema Por Unidade (pu), assim, tomou-se como base o valor de 100 MW para normalizar a carga.

O treinamento das ANNs ocorreram com o auxílio da função nftool do software Matlab. Para melhores resultados criou-se, para cada um desses arranjos, três ANNs que se diferenciam no número de neurônios na camada oculta. Isto é, foram criadas nove redes neurais a fim de analisar qual é o melhor número de neurônios em cada caso e, posteriormente, concluir qual teve um melhor desempenho. A entrada das redes neurais é um dos arranjos enquanto a saída é a carga total que o sistema de distribuição deve ter disponível. A Figura 5 apresenta o diagrama simplificado das entradas e saída das ANNs.

Figura 5. Diagrama simplificado do treinamento das ANNs.

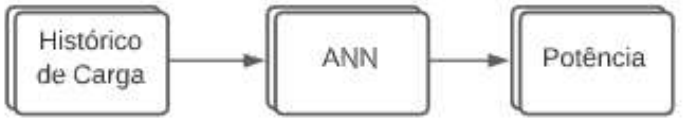

(a)

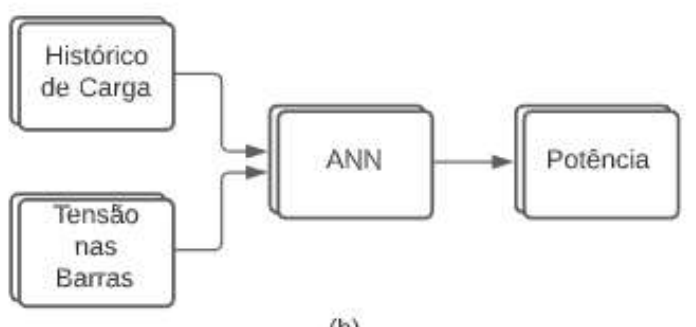

(b)

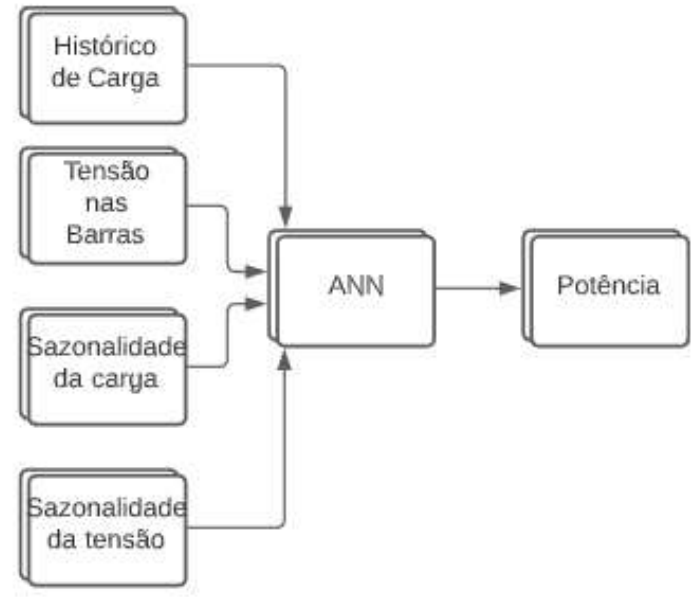

(c)

Fonte: Autores.

Utilizou-se de dados referentes à 20 dias anteriores para o treinamento das redes neurais artificiais, Tabela 2.

Tabela 2. Divisão do banco de dados.

\begin{tabular}{|c|c|}
\hline Banco de Dados & Período \\
\hline Treinamento & $01 / 05 / 2014$ a $20 / 05 / 2014$ \\
Previsão & $21 / 05 / 2014$ \\
\hline
\end{tabular}

Fonte: Autores.

No treinamento das ANNs há uma nova divisão dos dados, onde $70 \%$ foi utilizado para o treinamento de fato (estes são apresentados à rede durante o treinamento e a rede é ajustada de acordo com seu erro), 15\% para validação (estes são usados para medir a generalização da rede e interromper o treinamento quando a generalização para de melhorar) e $15 \%$ para testes (estes não afetam o treinamento e, apenas, fornecem uma medida independente do desempenho da rede durante e após o treinamento).

\section{Resultados e Discussão}

Com o objetivo de determinar quais dos arranjos gera uma ANN de melhor desempenho, aplicou-se o erro percentual médio absoluto (do Inglês, Mean Absolute Percentage Error - MAPE) e o erro quadrático médio (do Inglês, Mean Squared Error - MSE) como critério de avaliação.

O MSE mede a média dos quadrados dos erros, ou seja, a diferença quadrática média entre os valores estimados e o 
que é estimado. Os valores de MSE mais próximos de zero são melhores. O uso do erro quadrático médio leva em consideração o valor esperado da observação sendo previsto e elimina a arbitrariedade associada à soma residual dos quadrados. O MAPE é uma medida da precisão de um método de previsão em estatística, este expressa a precisão como uma porcentagem. Um valor de MAPE de 10\%, por exemplo, quer dizer que há um erro $10 \%$ na previsão dos dados.

Por tratar-se de previsão de curtíssimo prazo, escolheu-se realizar a previsão para até uma hora à frente como as amostras são feitas de 5 em 5 minutos, isto é, realizou-se a previsão para 12 instantes de tempo.

As Figuras 6, 7 e 8, apresentam graficamente os resultados obtidos pelas 9 redes neurais treinadas. Através da comparação entre a curva esperada e as curvas de cargas previstas, pode-se notar que a eficiência da metodologia proposta.

Figura 6. Representação gráfica do valor esperado e dos valores estimados pelo arranjo 1.

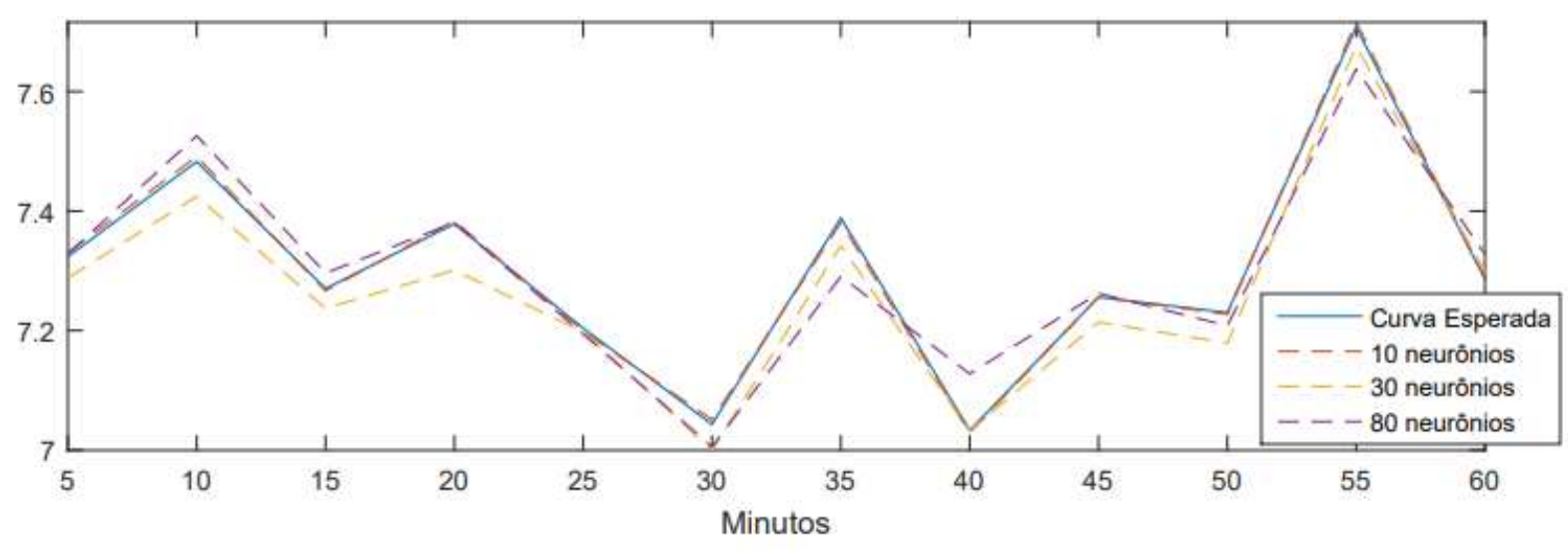

Fonte: Autores.

Figura 7. Representação gráfica do valor esperado e dos valores estimados pelo arranjo 2.

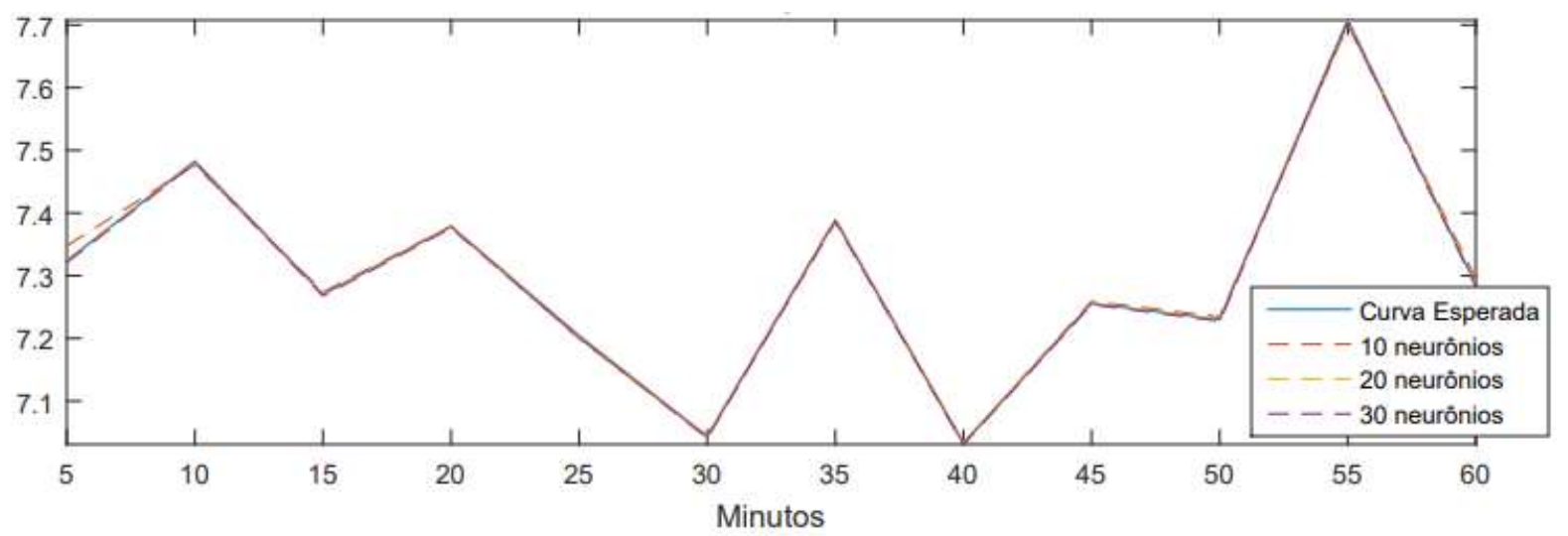

Fonte: Autores. 
Figura 8. Representação gráfica do valor esperado e dos valores estimados pelo arranjo 3.

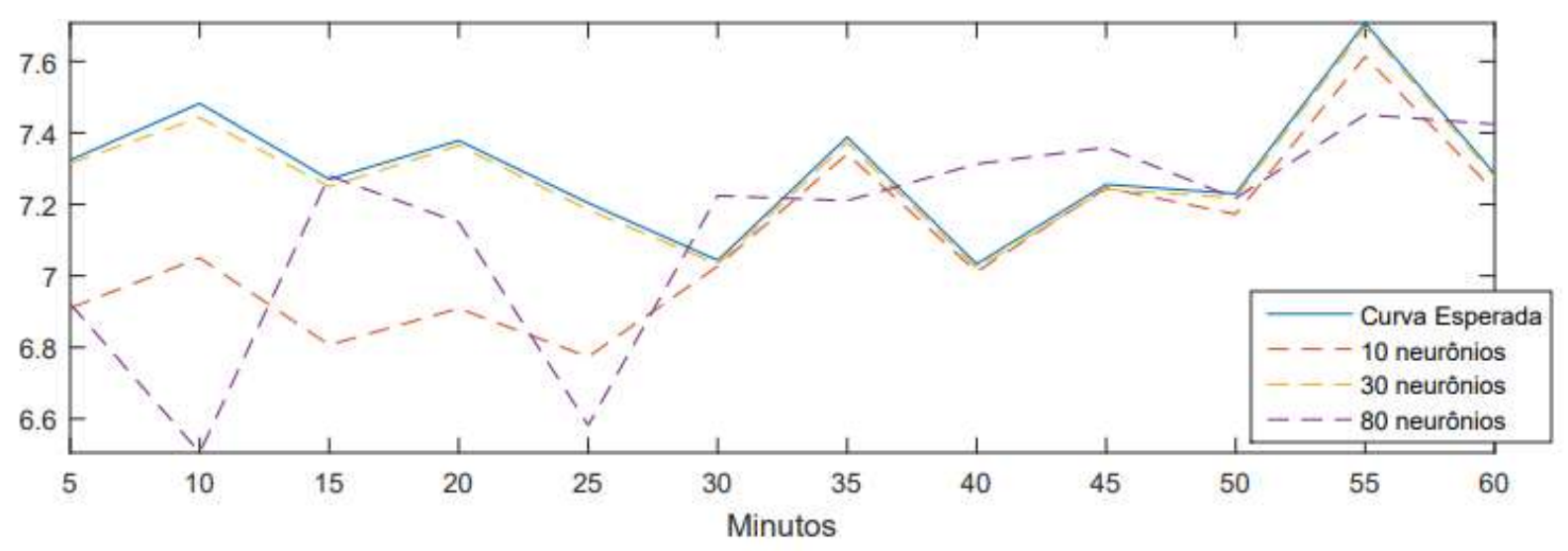

Fonte: Autores.

Nas Figuras 6 e 7 pode-se observar que as curvas da potência esperada e a prevista são bastante próximas, enquanto na Figura 8 a previsão com 10 e 80 neurônios nas camadas ocultas os resultados não são tão precisos. Assim, optou-se por fazer a análise detalhada apenas com uma das redes neurais de cada arranjo, a saber, a que apresentou melhor desempenho.

Dentre as nove redes neurais criadas, para o arranjo 1 a que apresentou melhor desempenho foi a com 10 neurônios na camada oculta, para o arranjo 2 foi a com 20 neurônios e para o arranjo 3 a ANN com 30 neurônios. Assim, a Tabela 3 traz o resultado esperado e o resultado da saída de cada uma dessas três redes neurais.

Tabela 3. Resultados da melhor combinação de neurônios de cada arranjo.

\begin{tabular}{|c|c|c|c|}
\hline Valor Esperado & Arranjo 1 & Arranjo 2 & Arranjo 3 \\
\hline 7,32380 & 7,33060151911128 & 7,32381896709747 & 7,31496858278407 \\
7,48240 & 7,4911186682630 & 7,48242410280248 & 7,44252721639211 \\
7,27020 & 7,26629478944918 & 7,27020073630154 & 7,24954504295353 \\
7,37860 & 7,38385048654906 & 7,37858201638172 & 7,36482744470074 \\
7,20390 & 7,19842619876665 & 7,20390644191478 & 7,18468599660976 \\
7,04320 & 7,05067576359072 & 7,04322010097870 & 7,03295156235243 \\
7,38830 & 7,38068766172341 & 7,38829384139609 & 7,37328120632544 \\
7,03290 & 7,02908911849289 & 7,03289560644146 & 7,02172451812309 \\
7,25590 & 7,25617669864917 & 7,25590490579877 & 7,24235147714355 \\
7,22990 & 7,22705778573581 & 7,22991981459005 & 7,21796973611781 \\
7,70760 & 7,71587470566113 & 7,70759278074974 & 7,69600713241443 \\
7,28560 & 7,28844794304640 & 7,28560303247296 & 7,27656880280954 \\
\hline
\end{tabular}

Fonte: Autores.

O cálculo dos parâmetros MSE e MAPE foi realizado através do software Matlab. O MSE com o uso da função immse do software Matlab e o MAPE por meio da equação a baixo, onde mean é a função que retorna o valor médio da matriz.

$$
M A P E=\operatorname{mean}\left(\frac{\text { Vetor dos Valores Reais }- \text { Valores Estimados pela ANN }}{\text { Valores Estimados pela ANN }}\right)
$$

Os resultados de MSE e MAPE são apresentados na Tabela 4. 
Tabela 4. MSE e MAPE obtidos.

\begin{tabular}{|c|c|c|}
\hline & MSE & MAPE \\
\cline { 1 - 1 } Arranjo 1 & $3,6858 \times 10^{-5}$ & $0,0719 \%$ \\
\cline { 1 - 1 } Arranjo 2 & $1,7471 \times 10^{-10}$ & $1,5271 \times 10^{-4} \%$ \\
\cline { 1 - 1 } Arranjo 3 & $2,8747 \times 10^{-4}$ & $0,2104 \%$ \\
\hline
\end{tabular}

Fonte: Autores.

Observa-se que os resultados alcançados são satisfatórios, com MAPE inferior a 1\% nos três arranjos comprovando assim um impacto econômico baixo conforme o estudo realizado em Ranaweera, Karady e Farner (1997) onde é colocado que a precisão da previsão de carga dentro de 5\% é adequada, considerando-se que uma inexatidão na previsão pode acarretar aumento no custo operacional do sistema.

Os algoritmos de ANN são muito dependentes dos dados (seja quantidade, características, granularidade e correlações). Assim, uma análise e manipulação criteriosas dessas características podem fornecer resultados distintos. Neste trabalho, foi possível verificar que as tensões nas barras de um sistema elétrico de potência é uma variável importante para a previsão de carga de curtíssimo prazo, uma vez que o valor real de muitas cargas varia de acordo com a tensão na qual as mesmas estejam sendo alimentadas.

Um fator que costuma ser muito impactante na previsão de cargas é a sazonalidade. Neste trabalho, a sazonalidade escolhida para estudo obteve resultados que atendem os pré-requisitos de erro máximo na previsão. Contudo, quando há a análise gráfica do resultado, nota-se que apenas o arranjo com 30 neurônios atinge uma boa previsão. Isso pode ser creditado ao tradeoff quantidade de entradas $\mathrm{x}$ complexidade das entradas.

Visto que os métodos baseados em inteligência artificial possuem a vantagem da não necessidade de uma complexa modelagem matemática para predição e uma de suas desvantagens é a difícil determinação de quais variáveis são importantes para um sistema de previsão, estudos como esse que fazem a avaliação quanto as variáveis são de grande importância para que se tenha previsões de demanda mais precisas.

\section{Considerações Finais}

Neste trabalho, foi proposta uma metodologia baseada em ANN levando em consideração como dados de entrada o histórico de consumo e tensões nas barras de cargas. Com a realização da comparação entre três arranjos de dados para treinamento de redes neurais e três arquiteturas de ANNs, foi possível apresentar o impacto da variável tensão de barra no resultado de previsão, mostrando assim que as tensões nas barras de um sistema elétrico de potência é uma variável valiosa quando se trata de previsão de carga. Outra variável impactante é a sazonalidade utilizada de histórico de carga. O arranjo que utilizou a tensão nas barras alcançou uma MAPE de $1,5271 \times 10^{-4} \%$.

É importante ressaltar que outros arranjos e otimizações das ANNs podem melhorar sensivelmente os resultados, dessa maneira sugere-se que novos modelos de redes neurais artificiais sejam desenvolvidos, com testes de diferentes arquiteturas e algoritmos de treinamento.

\section{Agradecimentos}

Os autores agradecem o Conselho Nacional de Desenvolvimento Científico e Tecnológico (CNPq) pelo apoio financeiro. 


\section{Referências}

Adeli, H., \& Yeh, C. (1989). Perceptron Learning in Engineering Design. Microcomputers in Civil Engineering. 4, 247-56. 10.1111/j.14678667.1989.tb00026.x

Almeida, V. (2013). Previsão de Carga através de Modelos Neuro-Fuzzy. 2013. 91 f. Bacharelado em Engenharia Elétrica - Universidade Federal do Rio de Janeiro - UFRJ

Amral, N., Ozvern, C. \& King, D. (2007). Short Term Load Forecasting using Multiple Linear Regression. In: IEEE International Universities Power Engineering Conference. 42. IEEE. 1192-8.

Antunes, J. Previsão de Carga Multinodal usando a Rede Neural ART-ARTMAP Fuzzy. 2013. 101 f. Tese (Doutorado em Engenharia Elétrica) - Universidade Estadual Paulista "Júlio de Mesquita Filho" UNESP, Ilha Solteira, 2013.

Baliyan A., Gaurav K., \& Mishra S. K., A. (2015). Review of Short Term Load Forecasting using Artificial Neural Network Models, Procedia Computer Science, 48, 121-125, https://doi.org/10.1016/j.procs.2015.04.160.

Box, G., \& Jenkins, G. (1976). Time Series Analysis: Forecasting and Control. ed. rev. Holden-Day.

Brito, N., Souza, B., Melo Filho, A., Lima, R., \& Pamplona, F. (2006). Previsão de Carga Utilizando Redes Neurais: Uma Aplicação ao Sistema CEAL. In: XVII Seminário Nacional De Distribuição De Energia Elétrica.

Caires, K. V. L., \& Simonelli, G. (2021). Previsão da demanda brasileira de biodiesel utilizando redes neurais artificiais . Research, Society and Development, 10(5), e17410513381. https://doi.org/10.33448/rsd-v10i5.13381.

Chafi Z. S., \& Afrakhte H. (2021). "Short-Term Load Forecasting Using Neural Network and Particle Swarm Optimization (PSO) Algorithm", Mathematical Problems in Engineering, vol. Article ID 5598267, 10 pages, 2021. https://doi.org/10.1155/2021/5598267

Chaika, P. Neural Networks. <https://www.poznavayka.org/en/science-and-technology/neural-networks/ >.

Cho, M., Hwang, J. \& Chen, C. (1995). Customer Short Term Load Forecasting by Using ARIMA Transfer Function Model. In: IEEE International Conference On Energy Management And Power Delivery. Singapore: IEEE. p. 317-322.

Christiaanse, W. (1971). Short-Term Load Forecasting Using General Exponential Smoothing. In: IEEE Transactions On Power Apparatus And SystemS. IEEE. p. 900-911.

Ehlers, R. S. (2005). Análise de Séries Temporais. 2005. 118 f. Relatório Técnico (Departamento de Estatística). Universidade Federal do Paraná - UFPR, Curitiba.

Estrela, C. (2018). Metodologia Científica: Ciência, Ensino, Pesquisa. Editora Artes Médicas.

Ettaouil, M., \& Ghanou, Y. (2009). Neural Architectures Optimization and Genetic Algorithms. In: WSEAS TRANSACTIONS ON COMPUTER. 8. p. 526537

Ferreira, A. (2020). Previsão De Carga Multinodal Utilizando Rede Neural ARTMAP Euclidiana. 2020. 76 f. Dissertação (Mestrado em Engenharia Elétrica) Universidade Estadual Paulista "Júlio de Mesquita Filho" UNESP, Ilha Solteira.

Guirelli, C. R. (2006). Previsão da Carga de Curto Prazo de Áreas Elétricas através de Técnicas de Inteligência Artificial. 2006.115 f. Tese (Doutorado em Engenharia) - Escola Politécnica, Universidade de São Paulo - USP, São Paulo.

Haykin, S. (1999). Redes Neurais: Princípios e Prática. (2a ed.), Bookman.

Highley, D. D., \& Hilmes, T. J. (1993). Load Forecasting by ANN. In: IEEE Computer Applications In Power. 3. IEEE. p. 10-15.

Hong, T., Gui, M., Baran, M., \& Willis, H. (2010). Modeling and Forecasting Hourly Electric Load by Multiple Linear Regression with Interactions. In: IEEE Pes General Meeting. Providence: IEEE. p. 1-8.

Kovàcs, Z. L. (1997). Redes Neurais Artificiais: Fundamentos e Aplicações. (2a ed.), Collegium Cognitio.

Kundur, P. (1994). Power System Stability and Control. McGraw-Hill.

Leal, A., Miguel, E., Baio, F., Neves, D., \& Leal, U. (2015). Redes Neurais Artificiais na Predição da Produtividade de Milho e Definição de Sítios de Manejo Diferenciado por Meio de Atributos do Solo. Bragantia, 74(4). 436-444.

Morettin, P., \& Toloi, C. (2006). Análise de Séries Temporais. (2a ed.), Blucher.

Oliveira, C. (2012). Previsão De Cargas Elétricas Através De Uma Rede Neural Híbrida Back-art Fuzzy. 2012.74 f. Dissertação (Mestrado em Engenharia Elétrica) - Universidade Estadual Paulista "Júlio de Mesquita Filho" UNESP, Ilha Solteira.

Pereira A. S. et al. (2018). Metodologia da pesquisa científica. UFSM.

Pessanha, J., \& Justino, T. (2013). Um Modelo para Previsão de Carga de Curto-prazo. Simpósio de excelência em gestão e tecnologia.

Pessoa, A. D., Sousa, G. C. L. Araujo, R. da C. de, \& Anjos, G. J. M. dos. (2021). Modelo de rede neural artificial para previsão da capacidade de carga de estacas cravadas. Research, Society and Development, 10(1), e12210111526. https://doi.org/10.33448/rsd-v10i1.11526 
Research, Society and Development, v. 10, n. 12, e600101220917, 2021

(CC BY 4.0) | ISSN 2525-3409 | DOI: http://dx.doi.org/10.33448/rsd-v10i12.20917

Ramchoun, H., Idrissi, M., Ghanou, Y., \& Ettaouil, M. (2015). Multilayer Perceptron: Architecture Optimization and Training. International Journal of Interactive Multimedia and Artificial Intelligence, 4(1).

Ranaweera D., Karady G., \& Farner R. (1997). Economic Impact Analysis of Load Forecasting. In: IEEE Transactions On Power Systems. IEEE. 1388-1392.

Shaikh, A. A project on Artificial Neural Network. Rizvi College of Engineering. https://www.slideshare.net/MohdArafatShaikh/artificial-neural-network80825958

Silva, I. (2010). Previsão de Vento para Geração de Energia Elétrica. 2010. Dissertação (Mestrado em Engenharia Elétrica) - Pontifícia Universidade Católica do Rio de Janeiro - PUC-RIO.

Singh S., Hussain S., \& BazazM. A. (2017). "Short term load forecasting using artificial neural network," 2017 Fourth International Conference on Image Information Processing (ICIIP), pp. 1-5, 10.1109/ICIIP.2017.8313703.

Taylor, J. (2012). Short-Term Load Forecasting with Exponentially Weighted Methods. In: IEEE Transactions On Power Systems. IEEE. p. $458-464$.

Vieira, R., \& Thomé, A. G. (2000). Avaliação de redes neurais aplicadas à previsão de índices de mercados de açães. Rio de Janeiro: NCE, Universidade Federal do Rio de Janeiro - UFRJ. Relatório Técnico. 6 p.

Zanini, A. (2004). Regulação Econômica no Setor Elétrico Brasileiro: Uma Metodologia para Definição de Fronteiras de Eficiência e Cálculo do Fator X para Empresas Distribuidoras de Energia Elétrica. 2004. 147 f. Tese (Doutorado em Engenharia Elétrica) - Pontifícia Universidade Católica do Rio de Janeiro - PUC-RIO. 